\title{
Standard Model gauge coupling unification
}

\author{
E.K. Loginov* \\ Ivanovo State University, \\ Ermaka St. 39, Ivanovo, 153025, Russia
}

November 9, 2018

\begin{abstract}
We study the low energy evolution of coupling constants of the standard model and show that gauge coupling unification can be achieved at the electroweak scale with a suitable normalization. We choose the grand unification group to be the semidirect product of $\operatorname{Spin}(8)$ by $S_{3}$. In this case the three low energy gauge couplings and the two scalar self-couplings are determined in terms of two independent parameters. In particular, it gives a precise prediction for the mass of the Higgs boson.
\end{abstract}

\section{Introduction}

The standard model (SM) is a mathematically consistent renormalizable field theory which predicts or is consistent with all experimental facts [1]. It successfully predicted the existence and form of the weak neutral current, the existence and masses of the $W$ and $Z$ bosons, and the charm quark, as necessitated by the GIM mechanism. The charged current weak interactions, as described by the generalized Fermi theory, were successfully incorporated, as was quantum electrodynamics. The consistency between theory and experiment indirectly tested the radiative corrections and ideas of renormalization and allowed the successful prediction of the top quark mass. Nevertheless,

${ }^{*}$ E-mail address: ek.loginov@mail.ru 
despite the apparent striking success of the theory, there are a lot of reasons why it is not the ultimate theory. First there is the well-established experimental observations of neutrino oscillations which are impossible in the SM. Secondly, some values of the SM parameters are not calculable in the theory, notably, the fermion mass hierarchy, the hierarchy of symmetrybreaking scales, and the Higgs boson mass. Hence the theory has far too much arbitrariness to be the final story. Finally, there exist purely theoretical difficulties in describing hadrons by means of the available methods of quantum field theory. These and other deficiencies of the SM motivated the effort to construct theories with higher unification of gauge symmetries.

In the framework of the grand unification hypothesis [2, 3], it is possible to obtain a reasonable explanation of the relation $\Lambda_{Q C D} \ll M_{G U T}$ that is based on the logarithmic renormalization-group dependence of the gauge coupling constant on the energy. Note, however, a similar analysis is not successful for the electroweak interaction, whose coupling constants are small at the scale $v \approx 246 \mathrm{GeV}$. It is unrelated to any dynamical scale and is introduced into the theory as a free parameter. One immediate consequence of the grand unification hypothesis is a very simple explanation for the experimentally observed charge quantization. This is because the eigenvalues of the generators of a simple non-Abelian group are discrete while those corresponding to the Abelian group are continuous. Unfortunately, by now LEP data have shown [4, 5, 6] that simple non-SUSY grand unifications must be excluded, initially by the increased accuracy in the measurement of the Weinberg angle, and by early bounds on the proton lifetime [7]. In other words, gauge coupling unification cannot be achieved in the SM if we choose the canonical normalization for the SM group, i.e., the Georgi-Glashow $S U(5)$ normalization. Also, to avoid proton decay induced by dimension- 6 operators via heavy gauge boson exchanges, the gauge coupling unification scale is constrained to be higher than about $5 \times 10^{15} \mathrm{GeV}$.

This latter restriction is not true for gauge-Higgs unification [8, 9, 10, 11], however. In gauge-Higgs models, the compactification scale may be of the order of the electroweak. Such unification is a very fascinating scenario beyond the SM since the Higgs doublet is identified with the extra component of the higher dimensional gauge field and its mass squared correction is predicted to be finite regardless of the non-renormalizable theory. This fact has opened a new possibility to solve the gauge hierarchy problem without, for example, supersymmetry. Obviously, the gauge-Higgs coupling unification can be achieved at the electroweak scale only with a suitable normalization. Note 
also that the unification group of the model does not necessarily be simple. For example, such group may be represented as a product of identical simple groups (with the same coupling constants by some discrete symmetries) or it may be obtained as an extension of a simple Lie group by means of a finite group of operators. The latter possibility will be considered in this paper.

\section{The $S_{3} \ltimes \operatorname{Spin}(8)$ symmetry}

We begin by discussing the following simple construction. This construction arises in connection with the following question: is it possible to embed an arbitrary group $G$ in some group $\widetilde{G}$ with the property that every automorphism of $G$ is the restriction of some inner automorphism of $\widetilde{G}$ ? Let $\Phi$ be a subgroup of Aut $G$. Then for $\widetilde{G}$ one may take the set of ordered pair $\phi g$ with multiplication defined by the rule

$$
\phi g \cdot \phi^{\prime} g^{\prime}=\phi \phi^{\prime} g^{\phi^{\prime}} g^{\prime}
$$

there $\phi \in \Phi$ and $g \in G$. (We are writing pairs without their customary comma and brackets.) The group axioms are straightforward to verify. From the rule for multiplication (11) it is immediate that $\phi^{-1} g \phi=g^{\phi}$. Hence the problem is solved. The group $\widetilde{G}$ is called the extension of the group $G$ by means of the automorphisms in $\Phi$ and denoted as $\Phi \ltimes G$. Alternatively one says that $\widetilde{G}$ is a semidirect product of $G$ by $\Phi$.

Now let $\Phi$ be a subgroup of the outer automorphisms group of $G$. Suppose $V$ is a representation space of $G$. Then the representation of $G$ in $V$ induces a representation of $\widetilde{G}$ in the direct sum $\widetilde{V}=V_{1} \oplus \cdots \oplus V_{n}$, where each direct summand $V_{i}$ is isomorphic to $V$ and $n=|\Phi|$. For the space $V_{i}$ one may take the ordered pair $\phi_{i} V$, where $\phi_{i} \in \Phi$. Then the action of $\widetilde{G}$ on $\widetilde{V}$ can be written as

$$
\phi g \cdot \oplus_{i} \phi_{i} V=\oplus_{i} \phi \phi_{i} g^{\phi_{i}} V .
$$

Let $G_{0}$ be a set of elements of $G$ such that $g^{\phi}=g$ for all $\phi \in \Phi$. Clearly, it is a $\Phi$-invariant subgroup of $G$. Using the formula (2) one may define a representation of $G_{0}$ on $\widetilde{V}$. It is easy to prove that $G_{0}$ acts equivalently on each direct summand of $\widetilde{V}$.

Let $G$ be a simple gauge group. If the normalization of the generators of $G$ are fixed, then the gauge couplings will be the same for both $G$ and $G_{0}$. Suppose that for the energy scale $\mu>M_{0}$ an $G$ gauge theory possesses 
both discrete and gauge symmetries, whereas for $\mu=M_{0}$ the symmetries breaking $\widetilde{G} \rightarrow G_{0}$ to take place. Then the representation space of $G_{0}$ reduces to $V$ and hence the normalization of the generators of $G_{0}$ must be changed. Since the definition of coupling constants depends on the normalization of the generators it follows that the gauge coupling of $G_{0}$ should be also change, namely $g^{2} \rightarrow g^{2}|\Phi|$ as $\widetilde{V} \rightarrow V$.

Now we suppose $G=\operatorname{Spin}(8)$. This group has the outer automorphisms group $S_{3}=\langle\rho, \sigma\rangle$, where $\rho^{3}=\sigma^{2}=(\rho \sigma)^{2}=1$, and two Majorana-Weyl real eght-dimensional representations that related to the eght-dimensional real vector representation by the action of $S_{3}$. The group $\operatorname{Spin}(8)$ cannot contain the SM group as a subgroup, but it contains disjoint subgroups $G_{3}$ and $G_{2} \times G_{1}$ that are isomorphic to $S U(3)$ and $S U(2) \times U(1)$, respectively. Moreover, we always can choose these subgroups in the following manner:

(i) $g^{\phi} \neq g$ for $1 \neq \phi \in S_{3}$ and $g \in G_{1}$,

(ii) $g^{\phi} \neq g=g^{\rho \sigma}$ for $1 \neq \phi \neq \rho \sigma$ and $g \in G_{2}$,

(iii) $g^{\phi}=g$ for all $\phi \in S_{3}$ and $g \in G_{3}$.

Hence, if for the energy scale $\mu=M_{0}$ the discrete and gauge symmetries breaking of $S_{3} \ltimes \operatorname{Spin}(8)$ to take place, then the gauge couplings of $G_{3}, G_{2}$, and $G_{1}$ should be satisfy

$$
g_{3}=\sqrt{3} g_{2}=\sqrt{6} g_{1}
$$

as $\mu=M_{0}$.

Just as for $\operatorname{Spin}(8)$, the group $S_{3} \ltimes \operatorname{Spin}(8)$ cannot contain the SM group as a subgroup. Nevertheless, in the next section we shall show that there is a way to break the symmetry by

$$
S_{3} \ltimes \operatorname{Spin}(8) \stackrel{S U(2)}{\longrightarrow} S U(3) \times U(1) .
$$

Moreover, the symmetry breaking is such that the gauge couplings of the SM group also satisfy (3). This possibility of the breaking is based on the following mathematical construction [12].

As was remarked before, the group $G$ admits the outer automorphisms $\rho$ and $\sigma$. Let

$$
G^{\sigma}=\left\{g \in G \mid g^{\sigma}=g\right\}
$$

(i.e. $G^{\sigma}$ is the centralizer of $\sigma$ in $G$ ). We denote by $S$ the factor space $G / G^{\sigma}$. Our nearest aim is to define a binary composition on the cosets of $G^{\sigma}$. Define

$$
L=\left\{g^{-1} g^{\rho \sigma} \mid g \in G\right\} .
$$


For each left coset $g G^{\sigma}$, there exists exactly one element $L_{a}$ in $L$ such that $L \cap g G^{\sigma}=\left\{L_{a}\right\}$. This defines a permutation representation of $G$ on $S$; if $g L_{a} G^{\sigma}=L_{b} G^{\sigma}$ for $g \in G$ let $g L_{a}=L_{b}$. Using this permutation representation, we may define a binary composition in $S$. If $L_{a} L_{b} G^{\sigma}=L_{c} G^{\sigma}$, define $a b=c$. This binary composition make $S$ into a nonassociative loop isomorphic to $\mathbb{S}^{7}$, which is defined in Appendix A. In particular, this defines the permutation representation of $G$ on $\mathbb{S}^{7}$. Extending this action by linearity on $\mathbb{O}$, we obtain the eight-dimensional spinor representation of $G$.

Apart from the left action of $G$ on $S$, there exists the trivial right action of $G$ on $S$ with $G^{\sigma}$ acting on itself by multiplication on the right. Suppose $G_{3} \subset G^{\sigma}$ and $\left(G_{2} \times G_{1}\right) \cap G^{\sigma}=1$. Then the (left ant right) actions of $G$ on $S$ induce the left action of $G_{2} \times G_{1}$ on $S$ and the right action of $G_{3}$ on $G^{\sigma}$. Obviously, these group actions are independent of each other.

\section{Gauge-Higgs unification}

In this section we briefly discuss the gauge-Higgs model based on the group $S_{3} \ltimes \operatorname{Spin}(8)$. Consider the $\operatorname{Spin}(8)$-invariant gauge theory defined on the manifold $M=M_{3,1} \times \mathbb{S}^{7}$, where $M_{3,1}$ is the Minkowski spacetime and $\mathbb{S}^{7}$ is the seven-dimensional sphere. (We assume that this sphere is equipped with the octonionic multiplication.) Suppose that this theory possess a symmetry under a discrete group $K$ of inner automorphisms. Further, let $A(x, y)$ and $C(x, y)$ be all gauge fields in the theory and only the fields $C(x, y) \in s o(7)_{v}$, where $s o(7)_{v}$ is the Lie algebra of $G^{\sigma}$. Following [13], we declare that only field configurations invariant under the action

$$
K:\left\{\begin{array}{l}
A(x, y) \rightarrow M(k) A\left(x, k^{-1}[y]\right) \\
C(x, y) \rightarrow N(k) C\left(x, k^{-1}[y]\right)
\end{array}\right.
$$

are physical. Here $M(k)$ and $N(k)$ are matrix representations of $K$ and $k[y]$ is the image of the point $y \in \mathbb{S}^{7}$ under the operation of $k \in K$. But unlike the standard orbifolding conditions, we suppose that $M(K) \neq N(K)$.

In order to determine automorphisms that are responsible for the symmetry breaking (4), we must first select the group $K$. Suppose $K=\mathbb{Z}_{4} \times \mathbb{Z}_{2}$. Further, suppose that the subgroup $H$ in $\operatorname{Spin}(8)$ is generated by the operators $I=R_{7}$ and $J=L_{7}^{-1} L_{5} L_{2}$, which are defined in Appendix B. Obviously, $I J=J I$ and $I^{4}=J^{2}=1$. It follows from this that $H=H_{I} \times H_{J}$, 
where the subgroups $H_{I}$ and $H_{J}$ are generated by $I$ and $J$, respectively, i.e., $H \simeq \mathbb{Z}_{4} \times \mathbb{Z}_{2}$. Define the action of $K$ on $\mathbb{S}^{7}$ and the representations $K \rightarrow M(K)$ and $K \rightarrow N(K)$ as follows. Let $f, f_{I}$, and $f_{J}$ be homomorphisms of $K$ onto $H, H_{I}$, and $H_{J}$ respectively. Then

$$
\begin{aligned}
M(k) A(x, y) & =f(k)^{-1} A(x, y) f(k), \\
N(k) C(x, y) & =f_{I}(k)^{-1} C(x, y) f_{I}(k), \\
k^{-1}[y] & =f_{J}(k)^{-1} y .
\end{aligned}
$$

We now focus our attention on the symmetry breaking at the fixed points. Since the factor group $K / K_{J}$ acts on $\mathbb{S}^{7}$ trivially, it follows that the $\operatorname{Spin}(8)$ gauge symmetry is reduced (under the given action) to the centralizer of $I$ in $\operatorname{Spin}(8)$, i.e., to $S U(4)_{s} \times U(1)$ (see also Appendix C). Therefore the fields $A(x, y)$ and $C(x, y)$ take its values in the Lie algebra $s u(4)_{s} \oplus u(1)$. In particular, $C(x, y)$ takes its values in $s u(3)_{s}$ (which is the intersection of $s u(4)_{s}$ and $\left.s o(7)_{v}\right)$. Now consider the $S U(4)_{s} \times U(1)$ symmetry breaking by $K_{J}$. It follows from (4) that the $S U(3)_{s}$ symmetry must be preserved under the action of $K_{J}$ on $\mathbb{S}^{7}$. This is possible only if the components of $C(x, y)$ are independent of $y$, i.e., if

$$
C_{M}(x, y)=i g_{s} C_{\mu}^{p}(x) \frac{\lambda_{p}}{2},
$$

where $\lambda_{p}$ are the usual Gell-Mann matrices for $S U(3)$ (cf. the formulas in Appendix C).

On the contrary, the unbroken symmetries of $A(x, y)$ must belong to the centralizer of $J$ in $S U(4)_{s} \times U(1)$. Using (B.2) and the explicit form of the generators of $S U(4)_{s} \times U(1)$, we prove that the fields $A(x, y)$ take its values in the Lie algebra $s u(2)_{s} \oplus u(1)$. Hence we can define these fields by

$$
\begin{aligned}
A_{M}(x) & =i g A_{\mu}^{k}(x) \frac{\sigma_{k}}{2}, \\
B_{M}(x, y) & =\left\{i g^{\prime} B_{\mu}(x), g^{\prime} \phi_{a}(x)\right\},
\end{aligned}
$$

where $\sigma_{k}$ are the standard Pauli matrices and $\phi_{a}$ are the components of $\phi(x) \in \mathbb{O}$. Strictly speaking, we would have to write $\phi(x) \in \mathbb{S}^{7}$. However, in this case the field $\phi(x)$ will be unobservable. The point is that for the KaluzaKlein type theories to be able to describe the observed four-dimensional world it is necessary for the extra spatial dimensions to be compactified down to a 
size which we do not probe in particle physics experiments (e.g. the Planck length). Therefore we suppose that the field $\phi(x)$ has quantum fluctuations such that $\phi(x) \in \mathbb{O}$ but not $\mathbb{S}^{7}$. In this case exactly one component of $\phi(x)$ (scalar field) will be observed.

We now consider the action of $K_{J}$ on $\mathbb{S}^{7}$. It is easily shown that the condition $J y=y$ is equivalent to $\left(e_{5}, e_{2}, y\right)=0$. Hence $y$ belong to an associative subalgebra of $\mathbb{O}$ generated by $e_{5}$ and $e_{2}$. Obviously, this is the algebra of quaternions with the basis $1, e_{2}, e_{5}, e_{7}$. The complexification of (1) transform this subalgebra into a two-dimensional unitary space. (Denote it by the symbol $\Phi$.) We have proved, in fact, that all nonzero fields $\phi(x)$ in (13) must belong to $\Phi$. Thus, if we identify $\phi(x)$ as the massless Higgs doublet, then we obtain a complete set of boson fields of the SM.

Finally, consider the SM Lagrangian for the field $\phi(x)$

$$
\mathcal{L}_{H}=\left(D_{\mu} \phi\right)^{\dagger}\left(D^{\mu} \phi\right)+\mu^{2} \phi^{2}-\lambda \phi^{4}
$$

and suppose that the fields $B_{\mu}(x)$ and $\phi_{a}(x)$ in (14) are components of $B(x, y)$. Then we have

$$
\lambda=g^{2} .
$$

As usual the Higgs vacuum expectation value breaks the $S U(2) \times U(1)$ symmetry down to $U(1)$. As a result we have the symmetry breaking (4).

\section{Gauge coupling unification}

The conditions (3) are valid in the $S_{3} \ltimes \operatorname{Spin}(8)$ limit. Now we need to study the regime $\mu<M_{0}$. The evolution of the SM gauge coupling constants in the one-loop approximation is controlled by the renormalization group equation

$$
\frac{d \alpha_{n}^{-1}(\mu)}{d \ln \mu}=\frac{b_{n}}{6 \pi}
$$

where $b_{1}=-2 n_{1}, b_{2}=22-2 n_{2}, b_{3}=33-2 n_{3}$, and $\alpha_{n}=g_{n}^{2} / 4 \pi$. (We have ignored the contribution coming from the Higgs scalar and higher-order effects.) It follows from (3) that the generators of SM group in the fundamental representation should be normalize by the condition $6 n_{3}=2 n_{2}=n_{1}=N_{f}$, where $N_{f}$ is the number of quark flavors. Expressing the low-energy cou-

plings in terms of more familiar parameters, we can represent the solutions 
of Eq. (16) as

$$
\begin{aligned}
\alpha_{s}^{-1}(\mu) & =\alpha_{3}^{-1}\left(M_{0}\right)-\frac{b_{3}}{6 \pi} \ln \frac{M_{0}}{\mu}, \\
\alpha^{-1}(\mu) \sin ^{2} \theta_{\mu} & =\alpha_{2}^{-1}\left(M_{0}\right)-\frac{b_{2}}{6 \pi} \ln \frac{M_{0}}{\mu}, \\
\frac{3}{5} \alpha^{-1}(\mu) \cos ^{2} \theta_{\mu} & =\alpha_{1}^{-1}\left(M_{0}\right)-\frac{b_{1}}{6 \pi} \ln \frac{M_{0}}{\mu} .
\end{aligned}
$$

Taking the linear combination [12 $\times$ Eq. (17) $-18 \times$ Eq. (18) $+7 \times$ Eq. (19)] and using the relations (3), we have

$$
\sin ^{2} \theta_{\mu}=\frac{7}{37}+\frac{60}{111} \frac{\alpha(\mu)}{\alpha_{s}(\mu)} .
$$

Obviously, Eq. (20) implies a non-trivial consistency condition among the gauge couplings. Taking the linear combination $[-78 \times$ Eq. (17) $+6 \times$ Eq. (18) + $10 \times$ Eq. (19)] $]$ and again using the relations (3), we have

$$
\ln \frac{M_{0}}{\mu}=\frac{6 \pi}{407}\left[\alpha^{-1}(\mu)-13 \alpha_{s}^{-1}(\mu)\right] .
$$

This determines the unification scale $M_{0}$. Also, combining Eqs. (20) and (21), we obtain

$$
\sin ^{2} \theta_{\mu}=\frac{3}{13}-\frac{110 \alpha(\mu)}{39 \pi} \ln \frac{M_{0}}{\mu} .
$$

Finally, it follows easily from Eqs. (17)-(19) that the running electroweak and strong gauge coupling constants satisfy

$$
\begin{aligned}
& \alpha^{-1}(\mu)=\alpha^{-1}\left(M_{0}\right)-\frac{66-13 N_{f}}{18 \pi} \ln \frac{M_{0}}{\mu}, \\
& \alpha_{s}^{-1}(\mu)=\alpha_{s}^{-1}\left(M_{0}\right)-\frac{99-N_{f}}{18 \pi} \ln \frac{M_{0}}{\mu},
\end{aligned}
$$

where the gauge couplings are connected by the relation $\alpha_{s}\left(M_{0}\right)=13 \alpha\left(M_{0}\right)$. Note that the choice of normalization of the generators essentially influences on the behavior of the gauge couplings by changing its values in fixed points. So for example Eqs. (23) and (24) will differ from that obtained in the SM. Therefore we must have a rule which permits to compare the gauge coupling 
constants in our (non-canonical) and the canonical normalizations. We will extract this rule from the renormalization on-shell scheme.

The on-shell scheme [14, 15, 16, 17, 18, 19] (see also Ref. [20]) promotes the tree-level formula $\sin ^{2} \theta_{W}=1-M_{W}^{2} / M_{Z}^{2}$ to a definition of the renormalized $\sin ^{2} \theta_{W}$ to all orders in perturbation theory, i.e.,

$$
\sin ^{2} \theta_{W}=\frac{\pi \alpha v^{2}}{M_{W}^{2}(1-\Delta r)}
$$

where $\Delta r$ summarizes the higher order terms. Here $\alpha$ is the fine structure constant, $M_{W}$ is the mass of the charged gauge boson, and $v=\left(\sqrt{2} G_{F}\right)^{-1 / 2}$ is the vacuum expectation value. One finds $\Delta r=\Delta r_{0}-\Delta r^{\prime}$, where $\Delta r_{0}=$ $1-\alpha / \alpha\left(M_{Z}\right)$ is due to the running of $\alpha$ and $\Delta r^{\prime}$ represents the top quark mass $m_{t}$ and the Higgs boson mass $M_{H}$ dependence. Using the formal expansion $\left(1+\Delta r^{\prime}\right)^{-1}=1-\Delta r^{\prime}+\ldots$, we can rewrite the formula (25) in the form

$$
\sin ^{2} \theta_{W}=\frac{\pi \alpha\left(M_{Z}\right) v^{2}}{M_{W}^{2}}\left(1-\frac{\alpha\left(M_{Z}\right)}{\alpha} \Delta r^{\prime}+\ldots\right) .
$$

In the on-shell scheme the value of $\sin ^{2} \theta_{W}$ is independent of the normalization of the generators. We suppose that the value of $\alpha(\mu)$ in the fixed point $\mu=M_{Z}$ is the same for both the canonical and non-canonical normalizations. Using this condition, we can now compare values of the running coupling constant $\alpha(\mu)$ in the two normalizations.

Following Ref. [21, 22, 23], we remove the $\left(m_{t}, M_{H}\right)$ dependent term from $\Delta r$ and write the renormalized $\sin ^{2} \theta_{\mu}$ (in the non-canonical normalizations) as

$$
\sin ^{2} \theta_{\mu}=\frac{\pi \alpha\left(\mu^{\prime}\right) v^{2}}{M_{W}^{2}}
$$

for $M_{Z} \leq \mu \leq \mu^{\prime} \leq v$. Further, we suppose that the unification scale coincides with the electroweak scale (i.e., $M_{0}=v$ ) and that the left-hand side of Eqs. (22) and (27) are equal as $\mu=M_{0}$. In this case

$$
\frac{M_{W}}{M_{0}}=\sqrt{\frac{13 \pi \alpha_{0}}{3}},
$$

where $\alpha_{0}=\alpha\left(M_{0}\right)$. It follows from (20)-(24) and (28) that the three parameters $\alpha\left(M_{Z}\right), \alpha_{s}\left(M_{Z}\right)$, and $\sin \theta_{W}$ of the SM are now determined in terms of one independent parameters $\alpha_{0}$. We show the gauge coupling unification in Fig. 1. Thus, there are two predictions. 


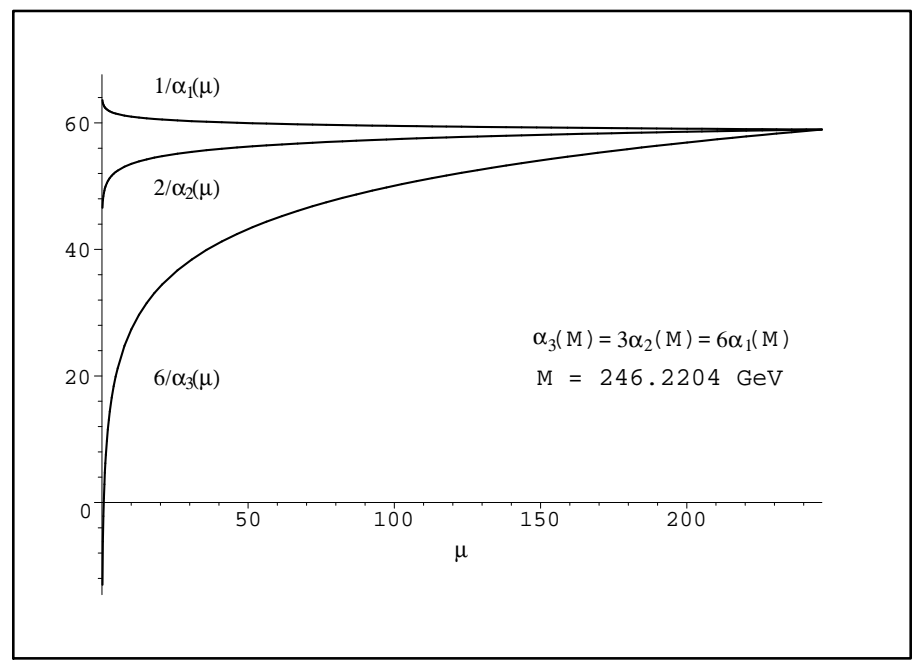

Figure 1: One-loop gauge coupling unification for the SM with the noncanonical normalization.

In conclusion, we show that the values of the coupling constants and the masses of the gauge bosons which are deduced from the SM are compatible with these predictions. Using $\alpha_{0}^{-1}=127.726$ and $N_{f}=6$ yield $\alpha^{-1}\left(M_{Z}\right)=127.937, \alpha_{s}\left(M_{Z}\right)=0.1221$, and $\sin ^{2} \theta_{W}=0.2229$. With more careful treatment of two-order effects, one obtains $\alpha_{s}\left(M_{Z}\right)=0.1210$. (Other parameters are changed unessentially.) These values are compatible with the SM predictions in Refs. [24, 25, 26, 27]. (For a recent review, see Ref. [1 and references therein.) This means, in particular that the value of $\alpha_{s}\left(M_{Z}\right)$ may also be chosen the same for both the canonical and non-canonical normalizations. Using $M_{0}=246.2204 \mathrm{GeV}$ yield $M_{W}=80.3841 \mathrm{GeV}$ and $M_{Z}=91.1876 \mathrm{GeV}$. This is also compatible with the SM predictions.

\section{$5 \quad$ Higgs boson mass}

The condition (15) gives a precise prediction about the Higgs mass $m_{\phi}$. Here we follow the presentation of Coleman and Weinberg [28] (see also [29]). The one-loop effective potential of $S U(2) \times U(1)$ gauge theory is given by

$$
V(\phi)=-\mu^{2} \phi^{2}+\lambda \phi^{4}+C \phi^{4} \ln \frac{\phi^{2}}{M^{2}}
$$


where $M$ is an arbitrary mass parameter and

$$
C=\frac{1}{16 \pi v^{4}}\left(3 \sum_{b} m_{b}^{4}+m_{\phi}^{4}-4 \sum_{f} m_{f}^{4}\right)
$$

Here the indices $b$ and $f$ run over the vector bosons and fermions (the top quark contribution is excluded), and the mass of the Higgs scalar is taken to zeroth order, i.e.,

$$
m_{\phi}^{2}=2 \mu^{2}=2 \lambda v^{2} .
$$

With (29), we can obtain the mass of the Higgs particle. It is given by

$$
m_{\phi}^{2}=2 v^{2}\left[\lambda+C\left(\ln \frac{v^{2}}{2 M^{2}}+\frac{3}{2}\right)\right] .
$$

We now use the condition (15). It follows from the formulas (3) and (28) that

$$
\lambda=\frac{3}{10} g^{2}=\frac{26 \pi \alpha_{0}}{5} .
$$

Substituting this expression into (31), one finds the mass of the Higgs scalar to zeroth order $\left(m_{\phi}=124.53 \mathrm{GeV}\right)$. Knowing the masses of the vector bosons and fermions, one may calculate $C$. (We obtain $C=0.0012$.) Finally, choosing the mass parameter $M=M_{Z}$, we have the Higgs boson mass $m_{\phi}=$ $126.15 \mathrm{GeV}$. This is agrees quite well with the experimental results that were recently obtained in [30, 31].

\section{Conclusion}

In this paper, we have shown that the gauge-Higgs model based on the group $S_{3} \ltimes \operatorname{Spin}(8)$ can be considered as a candidate for the real physical theory. It do not contradict SM (at least at the bosonic sector) and gives precise predictions for the masses of the gauge bosons and the Higgs scalar. Here we make two general remarks.

The group Spin(8) occupies a special position among the simple Lie groups since only it has outer automorphism group $S_{3}$. Namely this property of $\operatorname{Spin}(8)$ permits to define the non-canonical normalization in a natural way and to get the gauge coupling constants unification. To describe the $S_{3}$ symmetry breaking, we embedded the $\operatorname{Spin}(8)$-gauge theory in the model 
with larger global symmetry groups. The motivation for this is that whatever the high energy physics producing the spontaneous breaking of the gauge group, it is likely to possess a larger global symmetry than the gauged one. We risk to suppose that the existence of discrete $S_{3}$-symmetry is related to a duplication of the fermionic structure in the SM. We suppose that $S_{3} \ltimes K$ is the discrete flavor symmetry group. But of course this is only a hypothesis.

In order to describe the gauge-Higgs unification, we have used the relation between $\operatorname{Spin}(8)$ and the algebra of octonions. Generally speaking, this approach is not new. Properties of octonions was used earlier to describe various mechanisms of compactification of $d=11$ supergravity down to $d=4$ [32] (see also the review [33]) and to find solutions of the low-energy heterotic string theory [34]. There have been many other attempts over the years to incorporate this algebra into physics. The present paper is a next step in this direction.

\section{A Octonions}

We recall that the algebra of octonions $\mathbb{O}$ is a real linear algebra with the canonical basis $e_{0}=1, e_{1}, \ldots, e_{7}$ such that

$$
e_{i} e_{j}=-\delta_{i j}+c_{i j k} e_{k},
$$

where the structure constants $c_{i j k}$ are completely antisymmetric and nonzero and equal to unity for the seven combinations (or cycles)

$$
(i j k)=(123),(154),(167),(264),(275),(347),(365) .
$$

The algebra of octonions is not associative but alternative, i.e. the associator

$$
(x, y, z)=(x y) z-x(y z)
$$

is totally antisymmetric in $x, y, z$. Consequently, any two elements of $\mathbb{O}$ generate an associative subalgebra. The algebra (O) permits the involution (anti-automorphism of period two) $x \rightarrow \bar{x}$ such that the elements

$$
t(x)=x+\bar{x}, \quad n(x)=\bar{x} x
$$

are in $\mathbb{R}$. In the canonical basis, this involution is defined by $\bar{e}_{i}=-e_{i}$. It follows that the bilinear form

$$
(x, y)=\frac{1}{2}(\bar{x} y+\bar{y} x)
$$


is positive definite and defines an inner product on $\mathbb{O}$. It is easy to prove that the quadratic form $n(x)$ permits the composition

$$
n(x y)=n(x) n(y) .
$$

It follows from this that the seven-dimensional sphere

$$
\mathbb{S}^{7}=\{x \in \mathbb{O} \mid n(x)=1\}
$$

is closed relative to the multiplication in $\mathbb{O}$. Finally, since the quadratic form $n(x)$ is positive definite, it follows that $\mathbb{O}$ is a division algebra.

\section{B Triality}

Let $x$ be any element of $\mathbb{O}$. The left multiplications $L_{x}$ and right multiplications $R_{x}$ of $\mathbb{O}$ which are determined by $x$ are defined by

$$
L_{x} y=x y, \quad R_{x} y=y x
$$

for all $y$ in $\mathbb{O}$. Clearly $L_{x}$ and $R_{x}$ are linear operators on $\mathbb{O}$. We choose the canonical basis and denote by $L_{i}$ and $R_{i}$ the operators $L_{e_{i}}$ and $R_{e_{i}}$ respectively. Then from (A.1) and the fully antisymmetry of the associator (A.3), we get

$$
L_{i} L_{j}+L_{j} L_{i}=-2 \delta_{i j} I
$$

where $I$ is the identity $8 \times 8$ matrix. (Of course, a similar formula is true for the right multiplications.) Hence $L_{1}, \ldots, L_{7}$ are generators of the Clifford algebra $C l_{0,7}(\mathbb{R})$, and therefore they generate the Lie algebra $s o(8)$. This is the Lie multiplication algebra of $\mathbb{O}$.

In this algebra, we separate the subspaces $L$ spanned by the operators $L_{i}$ and the subalgebra $s o(7)_{s}$ spanned by the operators

$$
\begin{aligned}
S_{i} & =L_{i}+2 R_{i}, \\
D_{i j} & =L_{\left[e_{i}, e_{j}\right]}-R_{\left[e_{i}, e_{j}\right]}-3\left[L_{i}, R_{j}\right] .
\end{aligned}
$$

(The latter linearly generate the 14-dimensional exceptional simple Lie algebra $g_{2}$. ) This imply that the algebra $s o(8)$ decomposes into the direct sum

$$
s o(8)=s o(7)_{s} \oplus L
$$


The algebra so(8) admits the outer automorphisms $\rho$ and $\sigma$ of orders 3 and 2 respectively. We may define them by

$$
\left.\begin{array}{ll}
L_{i}^{\rho}=R_{i}, & R_{i}^{\rho}=-L_{i}-R_{i}, \\
L_{i}^{\sigma}=-R_{i}, & R_{i}^{\sigma}=-L_{i} .
\end{array}\right\}
$$

Obviously, the automorphisms $\rho \sigma, \sigma \rho$, and $\sigma$ fixe all elements of $s o(7)_{s}$, $s o(7)_{c}=s o(7)_{s}^{\rho}$, and $s o(7)_{v}=s o(7)_{s}^{\rho^{2}}$, respectively. The elements of intersection of the subalgebras, i.e. the elements of $g_{2}$, is fixed by $\rho$.

Just as for $s o(8)$, the group $\operatorname{Spin}(8)$ also admits the outer automorphisms $\rho$ and $\sigma$. According to (B.6), they are defined by

$$
\left.\begin{array}{ll}
L_{a}^{\rho}=R_{a}, & R_{a}^{\rho}=L_{a}^{-1} R_{a}^{-1} \\
L_{a}^{\sigma}=R_{a}^{-1}, & R_{a}^{\sigma}=L_{a}^{-1},
\end{array}\right\}
$$

where $a \in \mathbb{S}^{7}$. The automorphisms $\rho \sigma, \sigma \rho$, and $\sigma$ fixe the elements of $S O(7)_{s}$, $S O(7)_{c}=S O(7)_{s}^{\rho}$, and $S O(7)_{v}=S O(7)_{s}^{\rho^{2}}$ respectively. The intersection of the group, i.e. $G_{2}$, is fixed by $\rho$. Here $S O(7)_{v}$ is generated by the elements

$$
\begin{aligned}
R_{a}^{-1} L_{a} & =L_{a} R_{a}^{-1} \\
L_{a b}^{-1} L_{a} L_{b} & =R_{a b} R_{a}^{-1} R_{b}^{-1} .
\end{aligned}
$$

Note also that these automorphisms permute inequivalent irreducible representations $\mathbf{8}_{\mathbf{s}}, \mathbf{8}_{\mathbf{c}}$, and $\mathbf{8}_{\mathbf{v}}$ of the $\operatorname{Spin}(8)$ group having the same dimensionality.

\section{Complexification}

Suppose $\mathbb{C}$ is a subalgebra of $\mathbb{O}$ spanned by the elements 1 and $i=e_{7}$. We may consider $\mathbb{O}$ as a four dimensional complex (or rather unitary) space relative to the multiplication $a x$, where $a \in \mathbb{C}$ and $x \in \mathbb{O}$. This space is invariant under the unitary group, $S U(4)_{s} \times U(1)$, the Lie algebra of which decomposes into the direct sum

$$
s u(4)_{s} \oplus u(1)=s u(3)_{s} \oplus s u(2)_{s} \oplus u(1) \oplus V_{s}
$$

of the subspaces (but not the Lie subalgebras). We write down the generators of $S U(4)_{s} \times U(1)$ in the explicit form. 
1) $\mathrm{su}(3)_{\mathrm{s}}$ :

$$
\begin{aligned}
D_{53}-D_{42} & =6\left(e_{21}-e_{12}\right) \\
D_{15}-D_{26} & =6\left(e_{13}-e_{31}\right) \\
D_{31}-D_{64} & =6\left(e_{32}-e_{23}\right) \\
D_{23}-D_{54} & =6 i\left(e_{21}+e_{12}\right) \\
D_{65}-D_{12} & =6 i\left(e_{13}+e_{31}\right) \\
D_{41}-D_{36} & =6 i\left(e_{32}+e_{23}\right) \\
D_{16}-D_{34} & =6 i\left(e_{22}-e_{33}\right) \\
D_{52} & =2 i\left(e_{22}+e_{33}-2 e_{11}\right)
\end{aligned}
$$

2) $\mathbf{s u}(2)_{\mathbf{s}} \oplus \mathbf{u}(1)$ :

$$
\begin{aligned}
L_{2}+L_{7} L_{5} & =2\left(e_{10}-e_{01}\right) \\
L_{5}+L_{2} L_{7} & =2 i\left(e_{01}+e_{10}\right) \\
L_{7}+L_{5} L_{2} & =2 i\left(e_{00}-e_{11}\right) \\
R_{7} & =i\left(e_{00}+e_{11}+e_{22}+e_{33}\right)
\end{aligned}
$$

3) $\mathbf{V}_{\mathbf{s}}$ :

$$
\begin{aligned}
& L_{4}+L_{7} L_{3}=2\left(e_{20}-e_{02}\right) \\
& L_{6}+L_{7} L_{1}=2\left(e_{30}-e_{03}\right) \\
& L_{3}+L_{4} L_{7}=2 i\left(e_{02}+e_{20}\right) \\
& L_{1}+L_{6} L_{7}=2 i\left(e_{03}+e_{30}\right)
\end{aligned}
$$

Here $e_{i j}$ is the $4 \times 4$ matrix with $(i, j)$ th entry 1 , and all other entries 0 . Note that the automorphisms $\rho$ and $\sigma$ fixe the elements of $s u(3)_{s}$ and the automorphism $\rho \sigma$ fixes the elements of $s u(2)_{s}$ and $V_{s}$, while $R_{7} \in u(1)$ is not invariant under any element of $S_{3}$. Note also that $S U(4)_{s} \times U(1)$ is the centralizer of $R_{7}$ in $\operatorname{Spin}(8)$.

\section{References}

[1] J. Beringer et al. (Particle Data Group), Phys. Rev. D 86, 010001 (2012).

[2] H. Georgi and S.L. Glashow, Phys. Rev. Lett. 32, 438 (1974). 
[3] H. Georgi, H.R. Quinn, and S. Weinberg, Phys. Rev. Lett. 33, 451 (1974).

[4] P. Langacker and M.X. Luo, Phys. Rev. D 44, 817 (1991).

[5] J.R. Ellis, S. Kelley, and D.V. Nanopoulos, Phys. Lett. B 260, 131 (1991).

[6] U. Amaldi, W. de Boer, and H. Furstenau, Phys. Lett. B 260, 447 (1991).

[7] W.J. Marciano, Eighth Workshop on Grand Unification, ed. K. Wali, World Scientific Publishing Co., Singapore (1987).

[8] N.S. Manton, Nucl. Phys. B 158, 141 (1979).

[9] Y. Hosotani, Phys. Lett. B 126, 309 (1983).

[10] H. Hatanaka, T. Inami, and C.S. Lim, Mod. Phys. Lett. A 13, 2601 (1998).

[11] Y. Kawamura, Prog. Theor. Phys. 105, 999 (2001).

[12] S. Doro, Math. Proc. Camb. Phil. Soc. 83, 377 (1978).

[13] A. Hebecker and J. March-Russell, Nucl. Phys. B625, 128 (2002).

[14] A. Sirlin, Phys. Rev. D 22, 971 (1980).

[15] A. Sirlin, Phys. Rev. D 29, 89 (1984).

[16] D.C. Kennedy, B.W. Lynn, C.J.C. Im, and R.G. Stuart, Nucl. Phys. B 321, 83 (1989).

[17] D.C. Kennedy and B.W. Lynn, Nucl. Phys. B 322, 1 (1989).

[18] D.Yu. Bardin, M.S. Bilenky, G. Mitselmakher, T. Riemann, and M. Sachwitz, Z. Phys. C 44, 493 (1989).

[19] W. Hollik, Fortsch. Phys. 38, 165 (1990).

[20] Precision Tests of the Standard Electroweak Model, ed. P. Langacker (World Scientific, Singapore, 1995). 
[21] M.E. Peskin, T. Takeuchi, Phys. Rev. Lett. 65, 964 (1990).

[22] G. Altarelli, R. Barbieri, Phys. Lett. B 253, 161 (1991).

[23] V.A. Novikov, L.B. Okun, and M.I. Vysotsky, Nucl. Phys. B 397, 35 (1993).

[24] S. Schael et al., Phys. Reports 427, 257 (2006).

[25] P.A. Baikov, K.G. Chetyrkin, and J.H. Kuhn, Phys. Rev. Lett. 101, 012002 (2008).

[26] A.D. Martin, W.J. Stirlingb, R.S. Thornec, and G. Watta, Eur. Phys. J. C 64, 653 (2009).

[27] S. Bethke, Eur. Phys. J. C 64, 689 (2009).

[28] S. Coleman and E. Weinberg, Phys. Rev. D 7, 1888 (1973).

[29] T.P. Cheng and L.R. Li. Gauge Theory of Elementary Particle Physics. Oxford: Oxford University Press. 1984.

[30] G. Aad et al. [ATLAS Collaboration], Phys. Lett. B 716, 1 (2012).

[31] S. Chatrchyan et al. [CMS Collaboration], Phys. Lett. B 716, 30 (2012).

[32] F. Englert, Phys. Lett. B 119, 339 (1982).

[33] M.J. Duff, B.E.W. Nilsson, and C.N. Pope, Phys. Rept. 130, 1 (1986).

[34] J.A. Harvey and A. Strominger, Phys. Rev. Lett. 66, 549 (1991). 